\title{
Wir sollten auf Teambesprechungen verzichten
}

\author{
We Should Go Without Team Meetings
}

Autoren

Pro: Christian Kieser

Kontra: Wolfram Kawohl

Bibliografie

DOI http://dx.doi.org/

10.1055/s-0030-1266075

Psychiat Prax 2011; 38 :

111-113

(c) Georg Thieme Verlag KG

Stuttgart · New York .

ISSN 0303-4259

Korrespondenzadressen Dr. med. Christian Kieser Zentrum für Psychiatrie, Psychotherapie und Psychosomatik Klinikum Ernst von Bergmann In der Aue 59-61

14480 Potsdam

CKieser@klinikumevb.de

PD Dr. med. Wolfram Kawohl Psychiatrische Universitätsklinik Militärstraße 8

8004 Zürich, Schweiz

wolfram.kawohl@puk.zh.ch

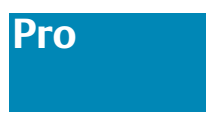

Regelmäßige Teambesprechungen haben sich im psychiatrischen Krankenhaus in den 1960er- und 1970er-Jahren als wichtiger Bestandteil psychiatrischer Praxis etabliert. Sie gewährleisten einen regelmäßigen Austausch zwischen Therapeuten und Pflegekräften, wobei u.a. Fragen der Diagnostik besprochen werden, die Behandlungsplanung im Team abgestimmt wird und Strategien im Umgang mit schwierigen Situationen entwickelt werden. Dennoch kann man sich fragen, ob die dafür aufgewandte Zeit tatsächlich optimal genutzt wird.

Jahrelange Praxis birgt die Gefahr, Inhalte und Ziele nicht mehr zu hinterfragen und als Routine im Wochenablauf einer Station durchzuführen. Teambesprechungen werden u.a. als gemeinsame „Klagemauer“ oder als „Kampffeld“ unausgesprochener Konflikte, zum „Kaffeeklatsch“ oder auch nach dem Motto „es war gut, wieder einmal darüber gesprochen zu haben“ genutzt. Für unzufriedene Mitarbeiter ist die Teambesprechung häufig ein willkommener Rahmen, ihren Unmut zum Ausdruck zu bringen, andere Mitarbeiter wiederum warten gelangweilt das Ende der Besprechung ab und erleben „das Team“ als Zeitvergeudung. Der Zeit- und Ressourcenaufwand ist schließlich erheblich. Bei 10 Teammitgliedern, die 1,5 Stunden zusammensitzen, ergibt das in der Summe 15 Stunden pro Woche entsprechend einer 0,375 VK einer Mitarbeiterstelle.

So ist auch unter Berücksichtigung neuerer Ansätze wie Recovery, Empowerment, shared decision making die Frage gerechtfertigt, ob die Teambesprechung konzeptionell weiterentwickelt werden kann bzw. welche Alternativen denkbar sind, um Zeit und Ressourcen bestmöglich für die therapeutische Arbeit mit den Patienten einzusetzen. Folgt man dem Grundsatz, nicht über Patienten, sondern mit den Patienten zu sprechen, sind Behandlungs- (BK) und Netzwerkkonferenzen (NK) eine mittlerweile bewährte Alternative zu den üblichen Teambesprechungen, die im Rahmen des „Need-adapted Treatment“ entwickelt wurden $[1,2]$.

\section{Behandlungs- und Netzwerkkonferenzen}

Bei der BK besprechen der Bezugstherapeut (in der Regel Arzt oder Psychologe) und ein Kotherapeut (Pflegekraft oder Sozialarbeiter) mit dem Patienten den Behandlungsauftrag, den Plan und das Ziel der Behandlung sowie die dazu erforderlichen Hilfen. Weitere Therapeuten sind als „Zuhörer“ mit anwesend. In der NK wird zusätzlich mit den Angehörigen und/oder professionellen Helfern (z.B. gesetzliche Betreuer, weiterbehandelnder Arzt, Sozialarbeiter des SPsD) und anderen wichtigen Bezugspersonen nach Lösungen gesucht. Die BK findet in der Regel einmal wöchentlich, die NK je nach Bedarf ein- bis mehrmals pro Aufenthalt statt.

Alle für den Patienten und seine Behandlung relevanten Aspekte werden in seiner Anwesenheit besprochen. Gegen Ende der BK bzw. NK wird das Reflecting-Team durchgeführt [3]. In Anwesenheit des Patienten - aber ohne direkt mit ihm zu sprechen - tauschen sich die bis dahin zuhörenden Therapeuten aus. Dazu gehören Überlegungen zum bisherigen Behandlungsverlauf, positive Konnotationen aber auch kritische Anmerkungen werden angesprochen, Chancen und Alternativen werden aufgezeigt. Nach der BK und NK wird nicht weiter über den Patienten gesprochen. Entsteht das Bedürfnis, einzelne Punkte zu vertiefen, die vor dem Patienten nicht erwähnt wurden, wird überlegt, was dies verhindert hat. Möglichkeiten werden erörtert, dies bei einer anderen passenden Gelegenheit wie z.B. Einzelgespräch, Gruppe oder nächste BK nachzuholen.

Das Konzept der BK bzw. NK geht auf die sog. Therapieversammlungen (treatment meetings) [4] zurück, die zeitgleich eine informative, diagnostische und therapeutische Funktion haben. Die BKs ersetzen die Visiten einschließlich der Oberarzt- 
und Chefarztvisiten. Ziel ist es, die Entscheidungen in Anwesenheit aller Beteiligten zu treffen. Ebenso erfolgt die Planung und Koordination der therapeutischen Abläufe im direkten Austausch mit dem Patienten. Größtmögliche Transparenz und gemeinsam getragene Entscheidungen sind Ziel des in den BKs und NKs praktizierten offenen Dialogs (open dialog) [5]. Zudem wird die Kompetenz der Mitarbeiter aus nichtärztlichen Berufsgruppen in Gesprächsführung und therapeutischen Interventionen intensiv gefördert. Teambesprechungen erübrigen sich in diesem Kontext, sind eher hinderlich, da sie nicht unmittelbar in den therapeutischen Prozess eingebunden und damit häufig schwer anschlussfähig sind.

\section{Erfahrungen mit Behandlungs- und Netzwerkkonferenzen}

Mit zunehmender Erfahrung in BKs und NKs nimmt das Bedürfnis der Therapeuten über Patienten in deren Abwesenheit zu sprechen ab. Es findet eine kontinuierliche Verschiebung auf Gespräche mit den Patienten statt. Die Scheu, kritische Inhalte in seiner Anwesenheit zu thematisieren, geht zurück. Mitarbeiter entwickeln eine respektvolle und angemessene Sprache, die es ermöglicht in Anwesenheit des Patienten Probleme und Konflikte offen zu benennen. Abfällige Bemerkungen bzw. entwertende Kommentare über Patienten kommen kaum vor. Insbesondere können durch das Reflecting-Team auch heikle Punkte berührt werden, ohne in eine verhärtete Konfrontation mit dem Patienten oder den Angehörigen zu geraten.

Sowohl Patienten als auch Angehörige beschreiben es als vertrauensfördernd, dass nicht „hinter ihrem Rücken“ gesprochen wird und Entscheidungen getroffen werden. Es entsteht eine Atmosphäre der Offenheit und Verlässlichkeit. Die Gefahr, dass im Team therapeutisch eher hinderliche „Geheimnisse“ über den Patienten und seiner Familie entstehen, ist gering. Indirekte Übermittlungswege von Entscheidungen (wie z.B. in der Teambesprechung vor 3 Tagen wurde entschieden, dass ...), die zu Verzerrungen führen können, werden vermieden. Häufige Wiederholungen von Inhalten und Überlegungen kommen kaum vor.

\section{Was ändert sich?}

Durch den Verzicht auf die Teambesprechung wird das Gespräch mit dem Patienten in der BK und NK zum zentralen Ort der therapeutischen Auseinandersetzung und damit Kernstück des Behandlungsprozesses. Wichtige Voraussetzungen sind, den Grundsatz „verhandeln statt behandeln“ ernst zu nehmen sowie eine Sprache zu verwenden, die wertschätzend und verbindlich ist. Im Blick zu behalten ist, nichts zu verschweigen, was für den therapeutischen Prozess wichtig ist.

Wenn statt Teambesprechungen BKs und NKs durchgeführt werden, steht vor allem mehr Zeit für das Gespräch mit den Patienten zur Verfügung. Ein gemeinsamer Suchprozess kommt in Gang, Ressourcen zu mobilisieren und Lösungen zu finden. Nicht mehr die Teambesprechung ist das Forum der Interpretationen, Bewertungen und Entscheidungen, die dem Patienten teilweise indirekt übermittelt werden, sondern die Auseinandersetzung wird direkt und auf Augenhöhe mit dem Patienten geführt.

Fazit

Auf Teambesprechungen zu verzichten, kann sich lohnen. Sowohl die Ressourcennutzung als auch die Gestaltung des therapeutischen Prozesses sprechen dafür. Behandlungs- und Netzwerkkonferenzen sind eine konkrete Alternative dazu.

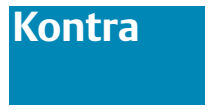

Keine Teambesprechungen mehr - zunächst ein verlockender Gedanke: Endlich mehr Zeit für Gespräche mit den Patienten und nicht nur über sie. Endlich mehr Zeit für die notwendigen, wenn auch meist ungeliebten, administrativen Aufgaben. Endlich selbst entscheiden können, wann und woher man sich die Informationen der anderen Teammitglieder holt, beispielsweise aus der Patientenakte oder der elektronischen Dokumentation. Wer hat dies nicht schon einmal erwogen? Doch halt - kann das wirklich gut gehen? Ist es sinnvoll, patientenbezogene Informationen nur noch aus der schriftlichen Dokumentation der anderen Teammitglieder zu entnehmen? Spätestens an diesem Punkt wird klar, dass eine Teambesprechung mehr ist als ein bloßer Austausch bereits dokumentierter Fakten. So wichtig die schriftliche Dokumentation von Behandlungsverläufen, diagnostischen Einschätzungen, Therapiemaßnahmen und Verhaltensbeobachtungen auch ist, die Reduktion auf nur einen Informationskanal ist in der Schriftform nicht zu vermeiden. Diese dürfte in den meisten Fällen nicht ausreichen, ein genügend detailliertes Bild des derzeitigen Zustandes sowie der Bedürfnislage der Patienten zu vermitteln. Außerdem verringern sich beispielsweise bei rein computergestützter Kommunikation nachweislich die Interaktionen zwischen den Teammitgliedern bei gleichzeitig zunehmenden Missverständnissen [6].

Zur Veranschaulichung der Problematik empfiehlt sich folgende Überlegung: Man stelle sich eine psychiatrische Intensiv- bzw. Akutstation vor, auf der Ärzte und Pflegepersonal nur noch schriftlich kommunizieren oder allenfalls kurze Statements zu dem von ihnen jeweils festgelegten weiteren Prozedere austauschen. Was in einer psychiatrischen Einheit der psychiatrischen Rehabilitation angesichts der resultierenden Missverständnisse eine schlechtere Behandlungsqualität zur Folge hätte, wäre hier mit Sicherheit früher oder später fatal. Feinheiten in der Zustandsbeschreibung eines Patienten lassen sich schriftlich oft nur unzureichend wiedergeben, ein Gesamtbild formt sich am ehesten durch die Möglichkeit des Nachfragens und der Kommunikation in einer bestimmten, nicht vorher festgelegten Interessensrichtung. Nicht zuletzt dienen Teambesprechungen auch dem gegenseitigen Lernen. Dies gilt über das Ausbildungsbedürfnis der in Weiterbildung befindlichen Assistenzärzte hinaus, der Austausch im multiprofessionellen Team mit seinen jeweiligen Spezialitäten kann in der nötigen Dichte nur im Rahmen von Besprechungen, die von Angesicht zu Angesicht und multilateral stattfinden, gewährleistet werden. Ärzte lernen so von Sozialarbeitern, Sozialarbeiter von Ärzten, beide vom Pflegepersonal und umgekehrt. Es besteht die Möglichkeit, nachzufragen wenn etwas unklar ist. Sich ergänzende Informationen fügen sich somit zu einem Gesamtbild, welches Zustand- und Bedürfnislage des Patienten möglichst umfassend abbildet. Die Festlegung eines gemeinsamen Vorgehens sowohl bezogen auf die einzelnen Patienten als auch generell bedarf eines regelmäßigen Austausches. Dabei gilt es einerseits, einen Verhaltenskodex zu entwickeln und zu pflegen, andererseits dienen solche Sitzungen auch dazu, eine gemeinsame Sprache im Babylon des psychiatrischen Teams [7], welches teilweise von unterschiedlichen Denkweisen und Terminologien geprägt ist [8], zu finden. Nicht von ungefähr gehören regelmäßige Teamsitzungen lt. Burns zu den Charakteristika gut funktionierender gemeindepsychiatrischer Einheiten [9]. 


\section{Anpassungsmöglichkeiten}

Der Ruf nach einer Abschaffung von Teambesprechungen ist also hochproblematisch. Gleichzeitig ist es verständlich, dass eine unkontrollierte Proliferation solcher Zeit beanspruchender Veranstaltungen, verhindert werden muss. Wie meistens macht auch hier die Dosis das Gift: Eine zeitliche Begrenzung von Teambesprechungen sowie eine Form, in der ein konstruktives Zusammenarbeiten möglich ist sowie eine Minimierung der Anzahl solcher Sitzungen erscheinen in der Tat ratsam. Statt nach einer Abschaffung zu rufen, sollte eher eine Optimierung bezüglich Zeit, Struktur und Häufigkeit angestrebt werden:

1. Für eine Station mit 20 Betten kann z. B. ein zunächst vielleicht streng erscheinendes Zeitlimit wie das Maximum von einer Dreiviertelstunde eingeführt werden.

2. Gleichzeitig sollten ablenkende Handlungen wie der Verzehr von Speisen und Getränken sollten vermieden werden, sodass eine wirkliche professionelle Arbeitsatmosphäre zustande kommen kann. Teamsitzungen dürfen auch nicht das Forum für vertiefende bzw. ausufernde Diskussionen sein [10]. Teamsitzungen, bei denen einer vorgegebenen Struktur entsprechend alle Patienten besprochen werden, tragen dazu bei, dies zu verhindern. Eine patientenbezogene Übergabe ist bei einem Schichtsystem allerdings unabdingbar. Ein ausschließliches Studium der schriftlichen Dokumentation kann auch hier nicht genügen, zumal häufig Ereignisse der jeweils vergangenen Stunden je nach Arbeitsaufkommen noch gar nicht dokumentiert werden konnten.

3. Teamsitzungen müssen regelmäßig durchgeführt werden und nicht nur dann, wenn ein akuter Bedarf empfunden wird. Die Intervalle dürften dabei zwischen verschiedenen Versorgungsformen differieren, sodass hier eine den jeweiligen Gegebenheiten angepasste Lösung gefunden werden muss. Ohne regelmäßige Teamsitzungen jedoch besteht die Gefahr, dass Patienten, die sich aufgrund ihrer Erkrankung oder ihrer Persönlichkeit eher im Hintergrund halten, zu wenig Beachtung des gesamten Teams finden.

\section{Fazit}

Zusammenfassend ist zu sagen, dass Teamsitzungen unter Voraussetzung einer vernünftigen Zeit- und Inhaltsstruktur und einer dem Bedarf angepassten Häufigkeit der Behandlungsqualität förderlich sind. Sie erweisen dem Zusammenhalt und der fachlichen Entwicklung des Teams ebenfalls einen guten Dienst. All dies steht letzten Endes im Interesse der Patienten.

\section{Literatur}

1 Aderhold V, Greve N. Was ist „Need-adapted Treatment“? Das Modell zur bedürfnisangepassten Behandlung von Menschen mit schizophrenen Psychosen. Soziale Psychiatrie 2004; 1: 1-8

2 Lehtinen K. Sozialpsychiatrie in Finnland. Das finnische Modell der Bedarfsorientierung. Soziale Psychiatrie 2001; 1: 23-25

3 Andersen T. Das Reflektierende Team. Dialoge und Dialoge über Dialoge. Dortmund: verlag modernes lernen; 1996

4 Alanen YO, Lehtinen $V$, Lehtinen $K$ et al. Das bedürfnisangepasste Behandlungsmodell - ein integriertes Modell zur Behandlung schizophrener und verwandter Psychosen. In: Aderhold V, Alanen Y, Hess G, Hohn P, Hrsg. Psychotherapie der Psychosen - Integrative Behandlungsansätze aus Skandinavien. Gießen: Psychosozial Verlag; 2003

5 Seikkula J. Offener Dialog mobilisiert selbst bei schwierigen Patienten die verborgenen Ressourcen. In: Greve N, Keller T, Hrsg. Systemische Praxis in der Psychiatrie. Heidelberg: Carl-Auer-Systeme Verlag; 2002

6 Tschannen D, Talsma A, Reinemeyer $N$ et al. Nursing Medication Administration and Workflow Using Computerized Physician Order Entry. Comput Inform Nurs 2010 [Epub ahead of print]

7 Rössler W. Psychologen in der psychiatrischen Klinik. Bonn: Psychiatrie-Verlag; 2004

8 Priebe S, Weih M, Kornhuber J. Ende der ärztlichen Dominanz in der Psychiatrie. Psychiat Prax 2010; 37: 216-218

9 Burns T. Team structure in community mental health. In: Morgan C, Bughra D, eds. Principles of Social Psychiatry; 2. ed. Chichester: Wiley-Blackwell; 2010

10 Burns T, O'Brien A. Assertive Case-Management und Case-Management in der Rehabilitation. In: Rössler W, ed. Psychiatrische Rehabilitation. Berlin Heidelberg: Springer; 2004 